\title{
Effect of Mechanical Stress on Lithiation and Sodiation Process
}

Hasti Asayesh-Ardakani ${ }^{1,3}$, Anmin Nie ${ }^{1,3}$, Yifei Yuan ${ }^{2,3}$ and Reza Shahbazian-Yassar 1, 2,3

1. Department of Mechanical Engineering-Engineering Mechanics, Michigan Technological University, Houghton, MI, USA

2. Department of Material Science and Engineering, Michigan Technological University, Houghton, MI, USA

3. Department of Mechanical and Industrial Engineering, University of Illinois at Chicago, Chicago, ILUSA

One of most challenging issues in batteries are performance and cyclability of batteries. The anode materials usually experience large volume changes through the ion insertion and extraction. This volume change and lithium embrittlement usually causes cracks and loss of contact in the anode material, which ultimately causes the failure of battery. On the other hand, the over-growing demand of high capacity storage for application in electrical vehicles and green power backup energy has triggered the new battery concept search beyond Li-ion. Na-ion rechargeable batteries are recent concepts. The natural abadency of $\mathrm{Na}$ on earth makes these batteries more eco-friendly and affordable.

Here, we investigated the structural change and reaction rates of anode material under stress during the $\mathrm{Li} / \mathrm{Na}$ insertion and how the mechanical properties of lithiated/sodiated material result in the battery performance. In this study, $\mathrm{ZnO}$ nanowires were chosen as anode material and investigation were carried out by using aberration corrected scanning transition electron microscopy and in situ TEM methods during sodiation and lithiation (Figure 1 shows a pristine $\mathrm{ZnO}$ nanowire and corresponding SAED pattern before and after lithiation). This direct comparison demonstrates the critical role of reaction rates and anode material's mechanical properties on failure mechanism and cyclability of Li/Na-ion batteries. The lithiation/sodiation of the material under stress causes non-uniform reaction rates. This produces dramatically reduce the capacity of battery. On the other hand, the lithiated $\mathrm{ZnO}$ nanowire shows the formation of cracks on the nanowires upon the first lithiation process, which shows low strength and ductility. This results in poor cyclability of battery. $\mathrm{ZnO}$ nanowires do not show any cracks and degradation after sodiation. Dislocations were observed on the surface of nanowire that results in more ductility of sodiated nanowire rather than lithiated one. 

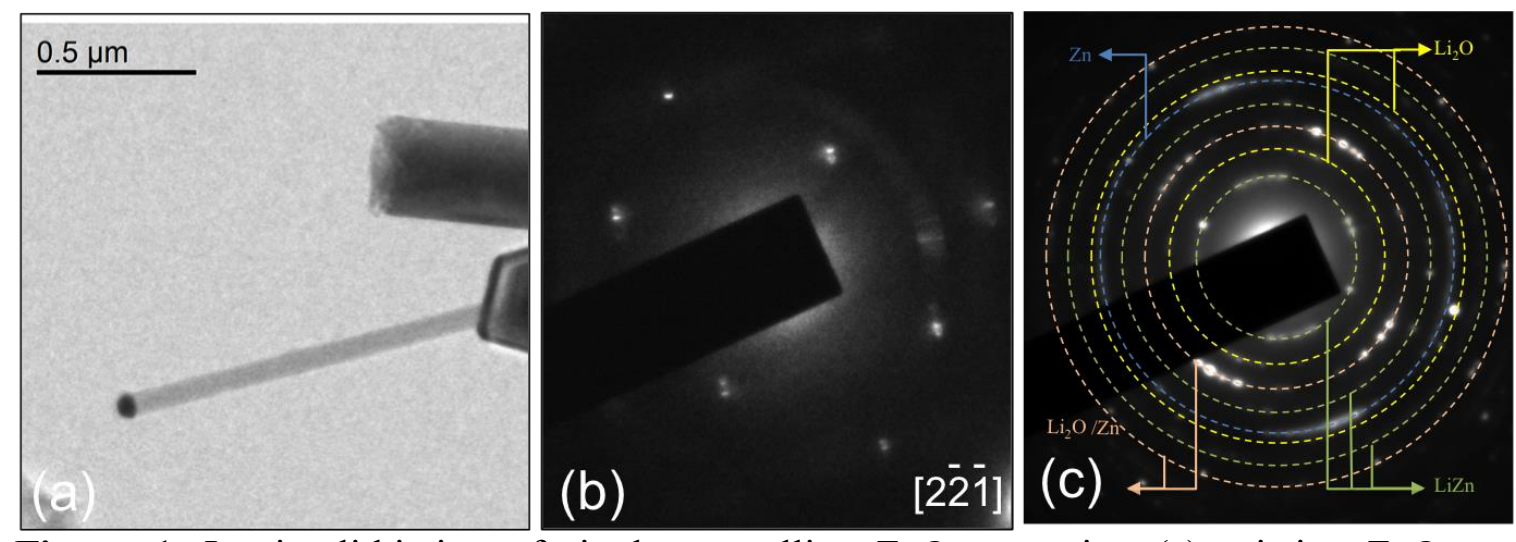

Figure 1. In situ lithiation of single crystalline $\mathrm{ZnO}$ nanowire. (a) pristine $\mathrm{ZnO}$ nanowire and (b) selected area electron diffraction pattern of pristine nanowire. (c) The selected area diffraction pattern of lithiated nanowire 